\title{
The Role of Combined ICRF and NBI Heating in JET Hybrid Plasmas in Quest for High D-T Fusion Yield
}

\author{
Mervi Mantsinen ${ }^{1,2, *}$, Clive Challis ${ }^{3}$, Domenico Frigione ${ }^{4}$, Jonathan Graves ${ }^{5}$, Joerg Hobirk ${ }^{6}$,Eva Belonohy ${ }^{6}$, Agata \\ Czarnecka $^{7}$, Jacob Eriksson ${ }^{8}$, Dani Gallart ${ }^{1}$, Marc Goniche ${ }^{9}$, Carl Hellesen ${ }^{8}$, Philippe Jacquet ${ }^{3}$, Emmanuel Joffrin ${ }^{9}$, \\ Damian King ${ }^{3}$, Natalia Krawczyk ${ }^{7}$, Morten Lennholm ${ }^{10}$, Ernesto Lerche ${ }^{11}$, Ewa Pawelec ${ }^{12}$, George Sips ${ }^{10}$, Emilia R. \\ Solano $^{13}$, Maximos Tsalas ${ }^{14}$, Marco Valisa ${ }^{15}$ and JET Contributors ${ }^{* *}$ \\ ${ }^{1}$ Barcelona Supercomputing Center (BSC), Barcelona, Spain \\ ${ }^{2}$ ICREA, Pg. Lluís Companys 23, 08010 Barcelona, Spain \\ ${ }^{3}$ CCFE, Culham Science Centre, Abingdon, Oxon, OX14 3DB, UK \\ ${ }^{4}$ ENEA, Fusion and Nuclear Safety Dep., C.R. Frascati, Frascati (Roma), Italy \\ ${ }^{5}$ Ecole Polytechnique Fédérale de Lausanne (EPFL), Swiss Plasma Center (SPC), CH-1015 Lausanne, Switzerland \\ ${ }^{6}$ Max-Planck-Institut für Plasmaphysik, D-85748 Garching, Germany \\ ${ }^{7}$ Institute of Plasma Physics and Laser Microfusion, Hery 23, 01-497 Warsaw, Poland \\ ${ }^{8}$ Uppsala University, SE-75120 Uppsala, Sweden \\ ${ }^{9}$ CEA, IRFM, F-13108 Saint Paul Lez Durance, France \\ ${ }^{10}$ European Commission, B-1049 Brussels, Belgium \\ ${ }^{11}$ Laboratory for Plasma Physics, LPP-ERM/KMS, Renaissancelaan 30 Avenue de la Renaissance B-1000, Brussels Belgium \\ ${ }^{12}$ Institute of Physics, Opole University, ul. Oleska 48, Opole 45-052, Poland \\ ${ }^{13}$ Laboratorio Nacional de Fusión, CIEMAT, Madrid, Spain \\ ${ }^{14}$ ITER Organization, Route de Vinon, CS 90 046, 13067 Saint Paul Lez Durance, France \\ ${ }^{15}$ Consorzio RFX, corso Stati Uniti 4, 35127 Padova, Italy
}

\begin{abstract}
Combined ICRF and NBI heating played a key role in achieving the world-record fusion yield in the first deuterium-tritium campaign at the JET tokamak in 1997. The current plans for JET include new experiments with deuterium-tritium (D-T) plasmas with more ITER-like conditions given the recently installed ITER-like wall (ILW). In the 2015-2016 campaigns, significant efforts have been devoted to the development of high-performance plasma scenarios compatible with ILW in preparation of the forthcoming D-T campaign. Good progress was made in both the inductive (baseline) and the hybrid scenario: a new record JET ILW fusion yield with a significantly extended duration of the high-performance phase was achieved. This paper reports on the progress with the hybrid scenario which is a candidate for ITER longpulse operation $(\sim 1000 \mathrm{~s})$ thanks to its improved normalized confinement, reduced plasma current and higher plasma beta with respect to the ITER reference baseline scenario. The combined NBI+ICRF power in the hybrid scenario was increased to $33 \mathrm{MW}$ and the record fusion yield, averaged over $100 \mathrm{~ms}$, to $2.9 \times 10^{16}$ neutrons/s from the 2014 ILW fusion record of $2.3 \times 10^{16}$ neutrons/s. Impurity control with ICRF waves was one of the key means for extending the duration of the high-performance phase. The main results are reviewed covering both key core and edge plasma issues.
\end{abstract}

\section{Introduction}

The application of ICRF waves in fusion plasmas is known to have a number of advantageous effects [1]. In addition to providing a fairly localised power deposition, the ICRF-driven fast ions arising through the resonant interactions between the ICRF waves and plasma ions can play a number of important roles for example by suppressing turbulence, enhancing the fusion reactivity and affecting MHD stability. In this paper, we report on the experiments carried out on the JET tokamak to optimise the use of ICRF waves in high-performance hybrid plasmas in preparation of the forthcoming D-T campaign. The optimization involves not only the selection of the ICRF scheme, the magnetic field and resonance location given the available ICRF frequencies but also the optimization of local gas puffing close to ICRF antennas for good ICRF coupling, optimization of the fuel ion mixture for optimal ICRF power partitioning and the choice of ICRF antenna phasing. Given that all of them affect the plasma performance in multiple ways, the optimization of the use of ICRF waves is an integral part of plasma scenario optimization and development. Its overall aim was to improve the fusion performance

\footnotetext{
Corresponding author: mervi.mantsinen@bsc.es

${ }^{* *}$ See the author list of X. Litaudon et al. Nucl. Fusion 57 (2017) 102001.
} 
and extend the duration of high-performance phase with respect to the 2014 record $2.5 \mathrm{MA} / 2.9 \mathrm{~T}$ hybrid discharge 86614 [2]. This discharge reached a normalised beta $\beta_{\mathrm{N}}$ of 2.1 , a neutron rate $\mathrm{R}_{\mathrm{NT}}$ of $2.3 \times 10^{16}$ neutrons/s and a confinement factor $\mathrm{H} 98(\mathrm{y}, 2)$ of 1.1. The goal for D-D fusion is $\mathrm{R}_{\mathrm{NT}} \approx 5 \times 10^{16}$ neutrons/s, to ensure a D-T fusion power of $\sim 15 \mathrm{MW}$. Combined ICRF and NBI heating in discharge 86614 was analysed in detail in [3]. In the present paper, experimental results on ICRF optimization in hybrid plasmas are presented while the results of ICRF+NBI modelling, including details on ICRF power partitioning and prediction for a 50\%:50\% D-T plasma, are reported in an accompanying paper [4].

Given the available ICRF frequencies and range of the toroidal magnetic field in JET, the ICRF schemes relevant for high-performance JET D-T plasmas are hydrogen $(\mathrm{H})$ and ${ }^{3} \mathrm{He}$ minority heating which coincide with second harmonic deuterium and tritium heating, respectively. The latter is the main ITER ICRF scheme for D-T plasmas and thus is of particular importance. However, all the experiments carried out in highperformance hybrid plasmas in the 2015-2016 JET experimental campaign were carried out with $\mathrm{H}$ minority heating. This was due to limited experimental time at high power together with operational restrictions on the ICRF use after ${ }^{3} \mathrm{He}$ puffing that are currently in force in JET.

Nevertheless, extensive computer modelling involving several ICRF codes were undertaken to prepare experiments with ${ }^{3} \mathrm{He}$ minority heating. The modelling results showed improved bulk ion heating and improved fusion performance with ${ }^{3} \mathrm{He}$ minority heating as compared to $\mathrm{H}$ minority heating $[5,6]$. Further beneficial effects on fusion performance are expected to arise from the stabilization of plasma microturbulence by ICRF-accelerated ${ }^{3} \mathrm{He}$ minority ions which was not included in the modelling.

In this paper we discuss the results obtained with $\mathrm{H}$ minority heating. They include the avoidance of impurity accumulation with ICRF waves and its dependence on the ICRF resonance location, the dependence of the fusion performance on the $\mathrm{H}$ minority concentration, and the achieved record fusion performance.

\section{ICRF waves for the avoidance of impurity accumulation}

Impurity control with ICRF waves was demonstrated as one of the key means for extending the duration of the high-performance phase in hybrid pulses. Figure 1 shows the main plasma parameters for two $1.5 \mathrm{MA} / 2.1 \mathrm{~T}$ hybrid discharges prepared in the same way except for the heating power waveforms. In discharge 90350 only NBI heating was applied while in discharge 90348 NBI power was reduced in the early part of the discharge and $2 \mathrm{MW}$ of ICRF heating was added at a frequency of 32 $\mathrm{MHz}$ for a central resonance.

As we can see from Fig 1, in the early phase of the discharges, up to $6 \mathrm{~s}$, discharges perform in the same way despite the fact that the total input power was about $10-20 \%$ lower in discharge 90348 with ICRF heating. However, from 6 s onwards, discharge 90350 with NBI heating only suffers impurity accumulation which leads to a deterioration of the fusion performance. In comparison, the performance is maintained throughout the heating phase in discharge 90348 with ICRF heating. The reason for this difference is believed to be the central heating provided by ICRF waves. It resulted in a more peaked electron temperature profile and a flatter density profile in the plasma core which were favourable for the avoidance of impurity accumulation. Our results are in line with previous results on ICRF heating for core impurity control, see .e.g. $[7,8,9]$ and references therein, extending them to high-performance hybrid plasmas of long duration for D-T with JET-ILW.

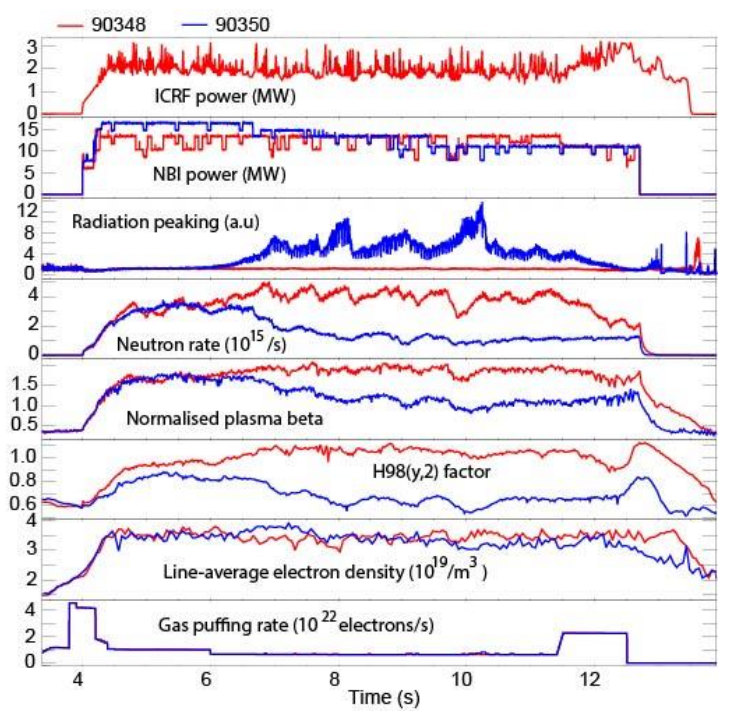

Fig. 1. Main plasma parameters for discharge 90348 with combined NBI and ICRF heating and for discharge 90350 with NBI heating only. The radiation peaking factor is the ratio of three most central bolometer channels to three channels offaxis and is an indication of the impurity content in the plasma center.

\section{Dependence of ICRF impurity control on the ICRF resonance location}

The dependence of impurity control on the ICRF resonance location was studied in plasmas with a normalised plasma beta $\beta_{\mathrm{N}}$ of about 2 and a safety factor profile optimized for MHD control. Figure 2 shows five hybrid discharges with $5 \mathrm{MW}$ of ICRF power at a frequency of $42 \mathrm{MHz}$ and up to $25 \mathrm{MW}$ of NBI power which were prepared in the same way except for the magnetic field $\mathrm{B}_{\mathrm{T}}$ and the start time of ICRF and NBI heating. $\mathrm{B}_{\mathrm{T}}$ was varied to change the ICRF resonance location from a high-field-side resonance at $\mathrm{R}_{\mathrm{res}} \approx 3.2 \mathrm{~m}$ to a low-field-side resonance at $\mathrm{R}_{\mathrm{res}} \approx 2.75 \mathrm{~m}$. The magnetic axis was located at $\mathrm{R}_{0} \approx 3.0 \mathrm{~m}$. Furthemore, the start time of ICRF and NBI heating was shifted by $0.25 \mathrm{~s}$ for each $\mathrm{B}_{\mathrm{T}}$ change of $0.1 \mathrm{~T}$ to keep the central safety factor as similar as possible at the heating onset. The $\mathrm{H}$ concentration $\mathrm{n}_{\mathrm{H}} /\left(\mathrm{n}_{\mathrm{H}}+\mathrm{n}_{\mathrm{D}}\right)$, as deduced from the ratio of the $\mathrm{D}_{\alpha}$ and $\mathrm{H}_{\alpha}$ light collected along lines of sight through the plasma, was $1-2 \%$. Penning gauge spectroscopy in the divertor gave somewhat higher $\mathrm{n}_{\mathrm{H}} /\left(\mathrm{n}_{\mathrm{H}}+\mathrm{n}_{\mathrm{D}}\right)$ of $3-4 \%$. 
The results shown in Fig. 2 suggest that a central resonance with $\left|R_{\text {res }}-R_{0}\right|<15 \mathrm{~cm}$ is favourable in order to prevent impurity accumulation and deleterious MHD in the present plasma conditions. It should be noted, however, that before the appearance of MHD and impurity accumulation discharge 92311 with a resonance furthest away on the high-field side produced similar or even better performance in terms of plasma energy content, $\beta_{\mathrm{N}}$, neutron rate $\mathrm{R}_{\mathrm{NT}}$ and electron and ion temperature as compared to discharges with a more central resonance. For this discharge, our modelling [4] predicts stronger direct fast wave electron damping until the onset of MHD activity. Unlike ion cyclotron damping, electron damping increases with the electron density and temperature and thus typically peaks in the plasma center. It could have played a role in the early phase of this discharge by increasing the central electron heating power deposition. This could be of interest if further ICRF optimization will be pursued e.g. with polychromatic operation.

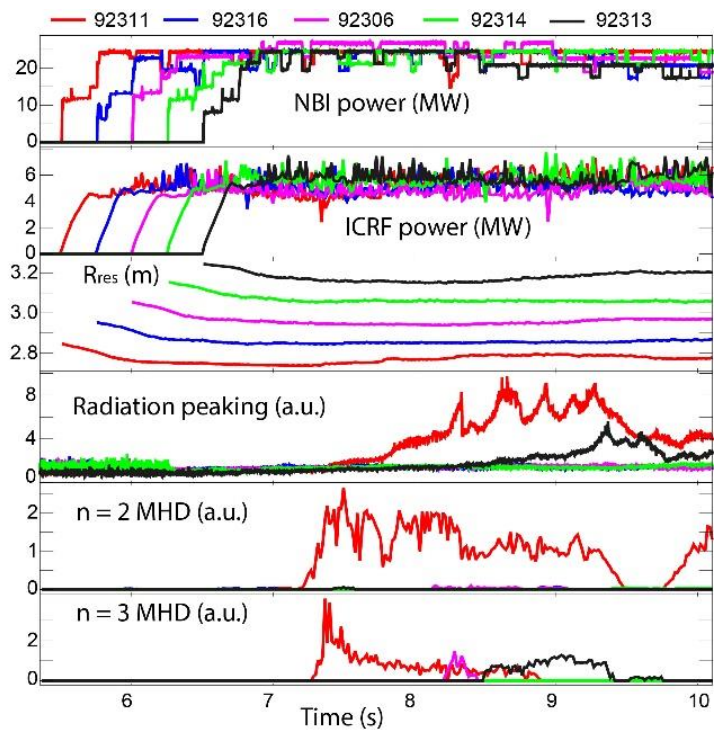

Fig. 2. Overview of five hybrid discharges with combined $\mathrm{NBI}+\mathrm{ICRF}$ heating at different toroidal magnetic fields to vary the ICRF resonance location: 2.6, 2.7, 2.8, 2.9 and $3.0 \mathrm{~T}$ in discharge 92311, 92316, 92306, 92314 and 92313, respectively. The start time of external heating was modified to match the central safety factor at the heating onset.

\section{Dependence of fusion performance on $\mathrm{H}$ minority concentration}

Another aim of the ICRF optimization in the recent 2015-2016 experiments was to clarify the role of the $\mathrm{H}$ concentration for fusion performance. The $\mathrm{H}$ concentration is known to play a key role for ICRF power partitioning between the $\mathrm{H}$ minority ions and the majority and beam-injected deuterium ions and thereby can affect the bulk plasma heating and the ICRF enhancement of $\mathrm{R}_{\mathrm{NT}}$ due to ICRF-accelerated deuterons [10].

Figure 3 shows the main plasma parameters for three $2.8 \mathrm{~T} / 2.4 \mathrm{MA}$ hybrid discharges with $5 \mathrm{MW}$ of ICRF power and $20 \mathrm{MW}$ of NBI power which were prepared in a same way except for $\mathrm{H}$ gas puffing. The resulting $\mathrm{n}_{\mathrm{H}} /\left(\mathrm{n}_{\mathrm{H}}+\mathrm{n}_{\mathrm{D}}\right)$ as deduced from the Penning gauge spectroscopy is approx. 3.5, 3 ad $6.5 \%$ in discharges 92321,92322 and 92323 , respectively. The ratio of the $\mathrm{D}_{\alpha}$ and $\mathrm{H}_{\alpha}$ light spectroscopy indicated similar relative changes but lower absolute $n_{H} /\left(n_{H}+n_{D}\right)$ values of $1.5-3$ $\%$.

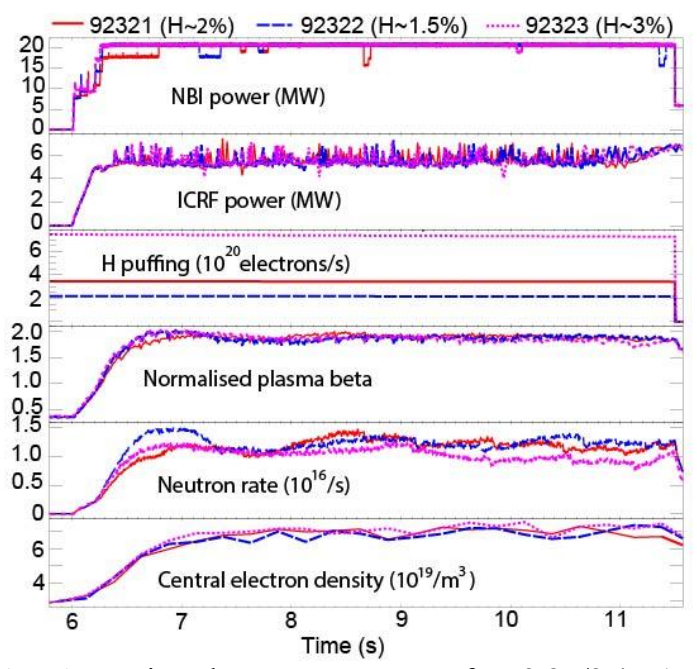

Fig. 3. Main plasma parameters for $2.8 \mathrm{~T} / 2.4 \mathrm{MA}$ hybrid discharges 92321, 92322 and 92323 with different $\mathrm{H}$ gas puffing rates to vary the $\mathrm{H}$ concentration.

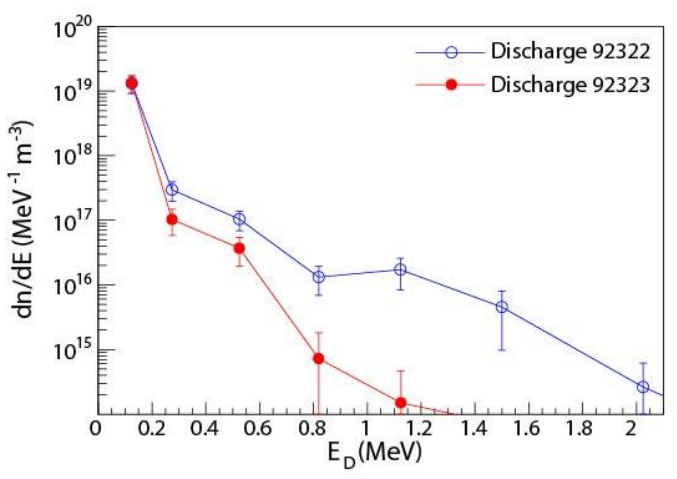

Fig. 4. D distribution functions as deduced from measurements with the neutron time-of-flight spectrometer TOFOR [11] for discharges 92322 and 92323 with a $\mathrm{H}$ concentration of $\sim 1.5 \%$ and $3 \%$, respectively.

The main difference between the discharges in the early part of the heating phase up to $t=8 \mathrm{~s}$ is the fast initial rise of $\mathrm{R}_{\mathrm{NT}}$ in the discharge with lowest $\mathrm{H}$ puffing. A lower $\mathrm{H}$ concentration in this discharge leads to a larger $\mathrm{D}$ damping and acceleration of $\mathrm{D}$ ions to higher energies than in other discharges. The difference in $R_{N T}$ reduces as the plasma density increases in time. Nevertheless, in the later steady phase of discharges from $\mathrm{t}=8 \mathrm{~s}$ onwards, $\mathrm{R}_{\mathrm{NT}}$ is $10-25 \%$ lower in discharge 92323 with the highest $\mathrm{H}$ concentration than in the discharges with lower $\mathrm{H}$ concentrations. This difference is consistent with a stronger ICRF-accelerated fast deuterium tail in discharges with lower $\mathrm{H}$ concentrations as deduced from the measurements with the neutron time-of-flight spectrometer TOFOR [11] (cf Fig. 4). It is also consistent with modelling which shows that central deuterium damping increases and hydrogen damping decreases when the $\mathrm{H}$ concentration is decreased while direct fast wave electron damping remains virtually the 
same [4]. We conclude that ICRF heating with a low $\mathrm{H}$ concentration is advantageous for maximising the D-D fusion yield through ICRF-acceleration of D ions while preventing impurity accumulation. The ICRF enhancement of $\mathrm{R}_{\mathrm{NT}}$ was typically $10-20 \%$ in the recent hybrid discharges [4]. Due to the different dependence of the D-T and D-D fusion cross section on the deuterium energy, the ICRF-enhancement of equivalent D-T fusion yield is smaller, of the order of $5 \%$ [4].

\section{Record fusion performance}

Figure 5 shows the main plasma parameters of the hybrid discharge with the highest fusion yield in the 2015-2016 experimental campaign. It was carried out at a plasma current of $2.2 \mathrm{MA}$ and a magnetic field of 2.8 T using $27 \mathrm{MW}$ of NBI heating and 4.5 MW of ICRF heating. The ICRF $\mathrm{H}$ minority resonance was in the plasma center and the $H$ concentration was $1-2 \%$ as deduced from the ratio of the $\mathrm{D}_{\alpha}$ and $\mathrm{H}_{\alpha}$ light. $\mathrm{R}_{\mathrm{NT}}, \beta_{\mathrm{N}}$ and $\mathrm{H} 98(\mathrm{y}, 2)$ steadily increased to their steady state values of $2.9 \times 10^{16} \mathrm{~s}^{-1}, 2.9$ and 1.2 , respectively. The radiated power increased slowly throughout the discharge and reached $12 \mathrm{MW}$ towards the end of the heating phase. The dominant impurities were mid-Z impurities $\mathrm{Ni}, \mathrm{Fe}, \mathrm{Cu}$ and Mo. At $\mathrm{t}=7.9 \mathrm{~s}$, the discharge was terminated by the machine protection system that detected a main chamber hot spot and a concomitant raise of the temperature of one of the poloidal limiters above the permitted limit of $940{ }^{\circ} \mathrm{C}$. The reason for the hot spot and the correct actions for its avoidance are still under investigation. In subsequent shots, the hot spot was mitigated with local gas puffing close to the limiter but it was not possible to progress further due to limited experimental time at the end of the campaign.

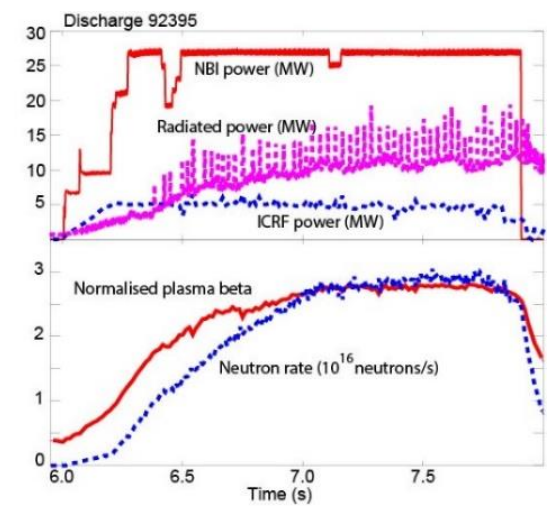

Fig. 5. Main plasma parameters for the 2016 record hybrid discharge 92395 with combined NBI + ICRF heating.

\section{Conclusions and future prospects}

Significant progress has been made in the development of the JET high-performance hybrid scenario compatible with the ITER-like wall in preparation of the future D-T campaign. A new record JET ILW fusion yield and an extended duration of the high-performance phase have been achieved. $\mathrm{H}$ minority heating with a central resonance and a low $\mathrm{H}$ minority concentration played key roles in avoiding central impurity accumulation and maximising the fusion performance and its duration.
Future plans include making use of a higher total ICRF+NBI power of $\sim 40 \mathrm{MW}$.

Three main topics remain for further optimization of combined ICRF and NBI heating in JET hybrid plasmas. First, ${ }^{3} \mathrm{He}$ minority heating needs to be experimentally demonstrated in high-performance D-T plasmas in preparation of the D-T campaign as discussed above. Second, the use of $+90^{\circ}$ degree ICRF phasing requires further experimental exploration. While all the experiments reported above used the dipole phasing of the JET A2 ICRF antennas, three hybrid discharges with $+90^{\circ}$ phasing of the A2 antennas were carried out. Figure 6 compares one of them with a similar discharge with dipole phasing. In broad terms similar plasma performance was obtained. However, one important difference was observed: the ICRF coupling resistance was larger by a factor of about two for all three discharges with $+90^{\circ}$ degree ICRF phasing as compared to all nine discharges with dipole phasing carried out in the same experimental session. This implies that it may be possible to increase ICRF power from the A2 antennas using $+90^{\circ}$ degree phasing, which would give important new prospects of increased input heating power and better fusion performance in JET D-T. Finally, the use of polychromatic ICRF operation may become relevant with high ICRF power at high $\beta_{\mathrm{N}}$.

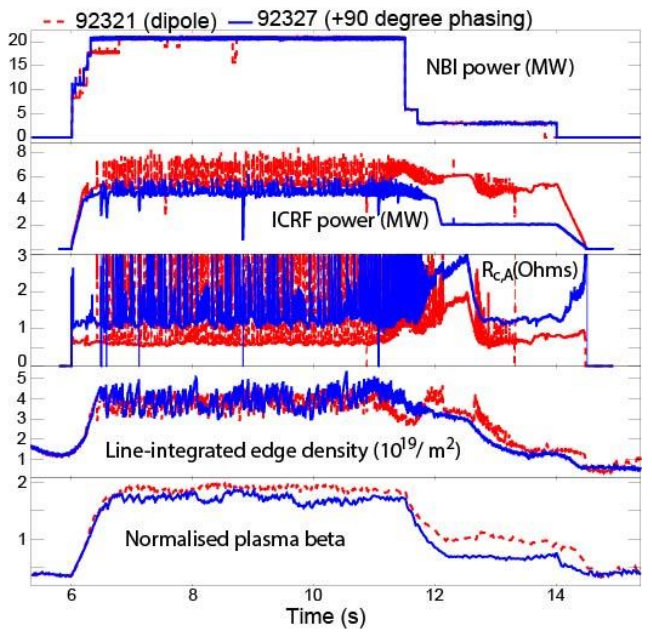

Fig. 6. NBI and ICRF power, coupling resistance for one of the A2 antennas, line-integrated edge plasma density and normalised beta for discharges 92321 and 92327 with dipole and $+90^{\circ}$ phasing of the A2 ICRF antennas, respectively.

This work has been carried out within the framework of the EUROfusion Consortium and has received funding from the Euratom research and training programme 2014-2018 under grant agreement No 633053. The views and opinions expressed herein do not necessarily reflect those of the European Commission.

\section{References}

1. M.J. Mantsinen et al., PPCF 45 (2003) A445 -A456.

2. Frigione et al., Europhysics Conference Abstracts 2015, vol. 39E, pp. P2.116.

3. M.J. Mantsinen et al., Europhysics Conference Abstracts 2015, vol. 39E, pp. P2.171.

4. D. Gallart et al., this conference.

5. D. Gallart et al., Europhysics Conference Abstracts 2016, Vol. 40A, P2.003. 
6. J. Garcia et al., PPCF 59 (2017) 014023.

7. C. Angioni et al., Nuclear Fusion 57 (2017) 056015.

8. M. Goniche et al., PPCF 59 (2017) 055001.

9. E. Lerche et al., Nuclear Fusion 56 (2016) 036022.

10. M.J. Mantsinen et al., PPCF 41 (1999) 843-865.

11. M. Gatu Johnson et al., Nucl. Instrum. Methods A, 591 (2008) 417. 